\title{
MEASURABLE FUNCTIONS ON HILBERT SPACE
}

\author{
BY \\ LEONARD GROSS(1)
}

1. Introduction. Consider a finite dimensional real vector space $L$, its dual space $L^{*}$ and a linear mapping $F$ from $L^{*}$ to the linear space $\mathscr{M}$ of all measurable functions modulo null functions on a probability measure space $(S, \mathscr{S}, \mu)$. If $y_{1}, \cdots, y_{n}$ is a basis of $L^{*}$ the set function $v_{0}$ defined on semi-infinite rectangles of $L$ by means of

$v_{0}\left(\left\{x \in L: y_{j}(x) \leqq \alpha_{j}, j=1, \cdots, n\right\}\right)=\mu\left(\left\{s \in S: F\left(y_{j}\right)(s) \leqq \alpha_{j}, \quad j=1, \cdots, n\right\}\right)$

extends in a well known way to a measure $v$ on the Borel sets of $L$, which is independent of the choice of basis $y_{1}, \cdots, y_{n}$ used in constructing it and depends only on the mapping $F$. Every probability Borel measure on $L$ arises in this way from such a linear mapping $F$ for if $\omega$ is a probability Borel measure then the elements of $L^{*}$ are measurable functions on the probability space $(L, \omega)$ and the identity map on $L^{*}$ fulfills the role of $F$, inducing as above a measure $v$ which may easily be seen to coincide with $\omega$. Clearly two such linear mappings $F$ and $F^{\prime}$ induce the same measure $v$ on $L$ if and only if for each finite set $y_{1}, \cdots, y_{k}$ of elements of $L^{*}$ (in particular for a basis of $L^{*}$ ) the random variables $F\left(y_{1}\right), \cdots, F\left(y_{k}\right)$ on the one hand and $F^{\prime}\left(y_{1}\right), \cdots, F^{\prime}\left(y_{k}\right)$ on the other hand have the same joint distribution in $k$-space.

This correspondence between linear mappings $F$ and probability measures $v$ on $L$ breaks down in case $L$ is not finite dimensional. If $L$ is, say, an infinite dimensional locally convex topological linear space then it is still true that a reasonable probability measure on $L$ gives rise to a linear mapping $F$ from the dual space $L^{*}$, of continuous linear functionals on $L$, to random variables, namely the identity mapping on $L^{*}$. However a given linear map from $L^{*}$ to random variables will not in general induce a measure on $L$ due to the absence of local compactness of $L$.

By a random variable on a probability space $(S, \mathscr{S}, \mu)$ we shall mean an element of the linear space of measurable functions modulo null functions on $S$.

Definition. A weak distribution on a topological linear space $L$ is an equivalence class of linear maps $F$ from $L^{*}$ to random variables on a probability space $(S, \mathscr{S}, \mu)$ (depending on $F$ ) where two such maps $F$ and $F^{\prime}$ are equivalent

Presented to the Society, October 31, 1959; received by the editors October 18, 1961.

(1) Most of the work on this paper was done while the author was an N.S.F. Fellow. 
if for each finite set $y_{1}, \cdots, y_{k}$ of vectors in $L^{*}, F\left(y_{1}\right), \cdots, F\left(y_{k}\right)$ have the same joint distribution in $k$-space as $F^{\prime}\left(y_{1}\right), \cdots, F^{\prime}\left(y_{k}\right)$.

This definition is due to I. E. Segal [7].

Thus a weak distribution on a finite dimensional space is equivalent to the notion of a measure on $L$ while on an infinite dimensional space it is a more general object than a measure on $L$. Nevertheless much of the usual integration theory in Euclidean spaces can be carried out in a modified form with respect to a weak distribution on an infinite dimensional space. See for example $[4 ; 5 ; 7 ; 8 ; 9]$. A tame function on $L$ is a function of the form $f(x)$ $=\phi\left(y_{1}(x), \cdots, y_{k}(x)\right)$ where $y_{1}, \cdots, y_{k}$ are in $L^{*}$ and $\phi$ is a Baire function on $E_{k}$. If $F$ is a representative of a weak distribution on $L$ and

$$
f(x)=\phi\left(y_{1}(x), \cdots, y_{k}(x)\right)
$$

is a same function on $L$ then $f^{\sim}=\phi\left(F\left(y_{1}\right), \cdots, F\left(y_{k}\right)\right)$ is a random variable and it is not hard to see that $f^{\sim}$ depends only on the function $f$ and on $F$ and not otherwise on the representation of $f$ in terms of $\phi$ and $y_{1}, \cdots, y_{k}$. The mapping $f \rightarrow f^{\sim}$ from the algebra of tame functions on $L$ to random variables is a homomorphism. It is not hard to verify that notions such as the integrability of $f^{\sim}$, the distribution of $f^{\sim}$ and the convergence in $L_{p}$ or in probability of sequences of such functions $f_{n}^{\sim}$ depend only on the weak distribution and not on the particular representative $F$. Thus for a given weak distribution we may consider questions about tame functions involving the above notions of integration theory. We remark that if $F$ is the identity map on $L^{*}$ then $f^{\sim}=f$. It is natural and for some purposes necessary to determine what other functions $g$ on $L$ besides tame functions can be made to correspond to random variables $g^{\sim}$ in such a way that the map $g \rightarrow g^{\sim}$ is an extension of that for tame functions and is linear and multiplicative while the above intrinsic integration notions such as the distribution, etc., of $g^{\sim}$ depends only on $g$ and the weak distribution and is independent of other arbitrary choices (such as representative of the weak distribution or of a basis of $L$ ).

It is the purpose of this paper to describe a class of continuous functions on $L$ for which such a correspondence with random variables can be established. We shall be concerned only with the case where $L$ is a real Hilbert space and the weak distribution is the canonical normal distribution with variance parameter $c$, i.e., that weak distribution which is uniquely determined by the condition that $F(y)$ is normally distributed with mean zero and variance $c\|y\|^{2}$. This weak distribution appears to be the best infinite dimensional analogue of Lebesgue measure in finite dimensional spaces. It is intimately related to the Wiener integral and has been found useful in studying certain operators and concepts that occur in quantum field theory. In two forthcoming papers we shall apply some of the results herein obtained to strengthen a theorem on non- 
linear transformations proved in [5] and to the study of harmonic analysis on a Hilbert space.

2. Measurable pseudo-norms. Let $H$ be a real Hilbert space. If $f$ is a tame function on $H$ then as noted in the Introduction the expectation and other intrinsic integration notions applied to the corresponding random variable $f^{\sim}$ does not depend on which representative of the given weak distribution is used to define $f^{\sim}$. Consequently in discussing the convergence in probability or distribution of random variables corresponding to tame functions it is not necessary to specify the representative of the given weak distribution. In fact we shall often leave out the tilde over tame functions $f$ in equations where it is clear that the corresponding random variable $f^{\sim}$ is actually meant. It is clear that a function on $H$ is a tame function if and only if there is a finite dimensional projection $P$ on $H$ such that $f(x)=f(P x)$ for all $x$ and such that $f$ restricted to the finite dimensional space $P H$ is a Baire function. $f$ is then said to be based on $P H$. If $f$ is based on $P H$ then it is clearly also based on $Q H$ whenever $Q \geqq P$ and $Q$ is a projection. If $f$ is based on the finite dimensional subspace $P H$ then we note that its expectation with respect to the canonical normal distribution with variance parameter $c$, which we restrict ourselves to exclusively, is given by

$$
E(\tilde{f})=(2 \pi c)^{-n 2} \int_{P H} f(x) \exp \left[-\|x\|^{2} / 2 c\right] d x
$$

when the integral exists where $n$ is the dimension of $P H$ and $d x$ is Lebesgue measure on $P H$. Finally we remark that a general existence and uniqueness theorem for weak distributions is given in [7] which ensures the existence and uniqueness of a weak distribution satisfying the defining relation for the normal distribution given above in the introduction.

Definition. A pseudo-norm $\|x\|_{1}$ on a real Hilbert space $H$ is called a measurable pseudo-norm if for every $\varepsilon>0$ there exists a finite dimensional projection $P_{0}$ such that for the tame function $\|P x\|_{1}$ we have

$$
\operatorname{Prob}(\|P x\| \tilde{i}>\varepsilon)<\varepsilon
$$

whenever $P$ is a finite dimensional projection orthogonal to $P_{0}$ where $\operatorname{Prob}\left(f^{\sim}>\varepsilon\right)$ refers to the distribution of the random variable $f^{\sim}$.

We shall generally denote pseudo-norms with a subscript.

REMARKS. (1) A pseudo-norm is always continuous on a finite dimensional space so that the function $\|P x\|_{1}$ is a tame function based on $P H$ and the condition in the definition makes sense.

(2) By applying equation (1) to the function $f(x)=\chi(\|P x\|)$ where $\chi$ is the characteristic function of $(\varepsilon, \infty)$ it may be seen by changing variables that $\operatorname{Prob}^{(c)}(\alpha\|P x\|>\varepsilon)=\operatorname{Prob}^{\left(\alpha^{2} c\right)}(\|P x\|>\varepsilon)$ where the superscript on Prob denotes the variance parameter. It follows easily that a pseudo-norm which is measurable 
with respect to the normal distribution with variance parameter $c$ is also measurable for any other variance parameter.

(3) It is easy to see that a positive multiple of a measurable pseudo-norm is also a measurable pseudo-norm as is also a sum of two measurable pseudonorms.

THEOREM 1. If $\|x\|_{1}$ is a measurable pseudo-norm on $H$ then the net $\|P x\|_{1}$ of tame functions converges in probability as $P$ approaches the identity operator through the directed set $\mathscr{F}$ of finite dimensional projections on $H .(\mathscr{F}$ is directed by inclusion of the ranges.) If $\|x\|_{1}$ denotes the limit random variable then for every $\varepsilon>0$ Prob $(\|x\| \tilde{i}<\varepsilon)>0$.

Proof. We may choose an increasing sequence of projections $P_{n}$ in $\mathscr{F}$ such that $\operatorname{Prob}\left(\|P x\|_{1}>1 / n\right)<1 / n$ whenever $P \perp P_{n}$ and $P$ is in $\mathscr{F}$. If $m>n>k$ then $\operatorname{Prob}\left(\left|\left\|P_{m} x\right\|_{1}-\left\|P_{n} x\right\|_{1}\right|>1 / k\right) \leqq \operatorname{Prob}\left(\left\|\left(P_{m}-P_{n}\right) x\right\|_{1}>1 / k\right) \leqq$ $\operatorname{Prob}\left(\left\|\left(P_{m}-P_{n}\right) x\right\|_{1}>1 / n\right) \leqq 1 / n$. Hence $\left\|P_{n} x\right\|_{1}$ converges in probability. Denote the limit function by $g$. Then if $P$ is in $\mathscr{F}$ and $P \geqq P_{n}$ we have for $k<n$

$$
\begin{aligned}
& \operatorname{Prob}\left(\left|g-\|P x\|_{1}\right|>1 / k\right) \\
& \leqq \operatorname{Prob}\left(\left|g-\left\|P_{n} x\right\|_{1}\right|>1 / k\right)+\operatorname{Prob}\left(\left|\left\|P_{n} x\right\|_{1}-\|P x\|_{1}\right|>1 / k\right) \\
& \leqq \operatorname{Prob}\left(\left|g-\left\|P_{n} x\right\|_{1}\right|>1 / k\right)+\operatorname{Prob}\left(\left\|\left(P-P_{n}\right) x\right\|_{1}>1 / n\right) \\
& \leqq \operatorname{Prob}\left(\left|g-\left\|P_{n} x\right\|_{1}\right|>1 / k\right)+1 / n .
\end{aligned}
$$

Hence not only the sequence $\left\|P_{n} x\right\|_{1}$ but also the whole net $\|P x\|_{1}$ converges in probability to $g$.

It remains to show that the essential lower bound of $g$ is zero. Now for any finite dimensional projection $P\left\{x \in P H:\|P x\|_{1}<\varepsilon / 2\right\}$ is a nonempty open convex set in the finite dimensional subspace $P H$ and therefore Prob $\left(\|P x\|_{1}<\varepsilon / 2\right)$ $>0$. Let $\varepsilon>0$ be a continuity point of the distribution function of $g$ and choose $n$ so large that $1 / n<\varepsilon / 2$ and we may also assume $n>2$. Let $k>n$. Since $\left\|\left(P_{k}-P_{n}\right) x\right\|_{1}$ and $\left\|P_{n} x\right\|_{1}$ are based on orthogonal subspaces they determine independent random variables. Hence

$$
\begin{aligned}
\operatorname{Prob}\left(\left\|P_{k} x\right\|_{1}<\varepsilon\right) & \geqq \operatorname{Prob}\left(\left\|\left(P_{k}-P_{n}\right) x\right\|_{1}+\left\|P_{n} x\right\|_{1}<\varepsilon\right) \\
& \geqq \operatorname{Prob}\left(\left\|\left(P_{k}-P_{n}\right) x\right\|_{1}<\varepsilon / 2,\left\|P_{n} x\right\|_{1}<\varepsilon / 2\right) \\
& \geqq \operatorname{Prob}\left(\left\|\left(P_{k}-P_{n}\right) x\right\|_{1}<\varepsilon / 2\right) \operatorname{Prob}\left(\left\|P_{n} x\right\|_{1}<\varepsilon / 2\right) \\
& \geqq 1 / 2 \operatorname{Prob}\left(\left\|P_{n} x\right\|_{1}<\varepsilon / 2\right) .
\end{aligned}
$$

Since $\operatorname{Prob}(g<\varepsilon)=\lim _{k \rightarrow \infty} \operatorname{Prob}\left(\left\|P_{k} x\right\|_{1}<\varepsilon\right)$ the theorem follows.

Notation. We denote by $H_{m}$ the topology on $H$ determined by the measurable pseudo-norms. By virtue of Remark(3) the basic open neighborhoods of zero are the unit spheres of measurable pseudo-norms. 
Definition. A function $f$ on $H$ is called uniformly continuous near zero in $H_{m}$ (u.c.n. 0 in $H_{m}$ ) if there exists a sequence $\|x\|_{n}$ of measurable pseudo-norms such that $\|x\|_{n}$ converges to zero in probability while $f$ is uniformly continuous in the topology $H_{m}$ on the unit sphere of $\|x\|_{n}$ for each $n$.

REMARK. If $H$ is finite dimensional then the preceding definition is equivalent to ordinary continuity since the Euclidean norm is measurable (so that $H_{m}$ is the ordinary topology) and a continuous function is uniformly continuous on compact sets.

THEOREM 2. If $f$ is a complex valued function which is u.c.n. 0 in $H_{m}$ then the net $f(P x)$ of tame functions converges in probability as $P$ approaches the identity through the directed set $\mathscr{F}$ of finite dimensional projections.

Proof. The proof is similar to the proof of Theorem 1 of [5]. Given $\varepsilon>0$ there exists a measurable pseudo-norm $\|x\|_{1}$ such that $\operatorname{Prob}(\|x\| \tilde{i}>1 / 2)$ $<\varepsilon / 3$ and such that $f$ is uniformly continuous in the topology $H_{m}$ on the set $S=\left\{x:\|x\|_{1} \leqq 1\right\}$. There exists a measurable pseudo-norm $\|x\|_{2}$ such that $|f(x)-f(y)|<\varepsilon$ whenever $x$ and $y$ are in $S$ and $\|x-y\|_{2}<1$. Let $P_{0}$ be such a large finite dimensional projection that if $P$ is in $\mathscr{F}$ and $P \geqq P_{0}$ then Prob $\left(\|P x\|_{1}>1\right)<\varepsilon / 3$ while at the same time $Q \perp P_{0}$ implies $\operatorname{Prob}\left(\|Q x\|_{2}>1 / 2\right)<\varepsilon / 6$ for $Q$ in $\mathscr{F}$. Now suppose that $P$ and $Q$ are in $\mathscr{F}$ and $P \geqq P_{0}$ and $Q \geqq P_{0}$. Since $\|(P-Q) x\|_{2} \leqq\left\|\left(P-P_{0}\right) x\right\|_{2}+\left\|\left(Q-P_{0}\right) x\right\|_{2}$ we have

$$
\begin{gathered}
\left\{x:\|(P-Q) x\|_{2} \leqq 1\right\} \supset\left\{x:\left\|\left(P-P_{0}\right) x\right\|_{2} \leqq 1 / 2\right\} \\
\cap\left\{x:\left\|\left(Q-P_{0}\right) x\right\|_{2} \leqq 1 / 2\right\}
\end{gathered}
$$

and $\operatorname{Prob}\left(\|(P-Q) x\|_{2} \leqq 1\right) \geqq 1-\varepsilon / 3$. But $|f(P x)-f(Q x)|<\varepsilon$ whenever $\|P x\|_{1} \leqq 1$, $\|Q x\|_{1} \leqq 1$ and $\|(P-Q) x\|_{2} \leqq 1$. Since each of these three conditions holds with probability at least $1-\varepsilon / 3$ they hold simultaneously with probability at least $1-\varepsilon$. Hence $\operatorname{Prob}(|f(P x)-f(Q x)| \geqq \varepsilon) \leqq \varepsilon$ whenever $P$ and $Q$ bound $P_{0}$. Now let $\varepsilon=1 / n$ and denote by $P_{n}$ the projection $P_{0}$ obtained above for a general $\varepsilon$. The $P_{n}$ may with no loss of generality be assumed increasing. Then $f\left(P_{n} x\right)$ converges in probability to a function $f^{\sim}$ and as in the proof of Theorem 1 it may be seen that the whole net $\{f \circ P\}$ converges in probability to $f^{\sim}$.

In the following section we shall give some examples of measurable pseudonorms which may be simply defined in an abstract Hilbert space. In order to investigate more deeply the relation between the normal distribution and the Wiener integral it is useful to establish the measurability of certain pseudo-norms on $L^{2}(0,1)$ (see $\left.\S 5\right)$. To this end it is convenient to prove first some convergence theorems for measurable pseudo-norms. This we shall do in $\$ 4$.

3. A class of measurable pseudo-norms. Under suitable restrictions on a homogeneous positive polynomial $p\left(t_{1}, \cdots, t_{k}\right)$ of degree $2 n$ in $k$ real variables its 
$2 n$th root defines a pseudo-norm on $E_{k}$. In this section we describe these polynomials and their analogues on a real Hilbert space and obtain from among them some measurable pseudo-norms.

It is convenient to use the terminology of tensors instead of polynomials. Let $H$ be a real Hilbert space. We shall denote by $H^{(n)}$ the Hilbert space of symmetric $n$-tensors over $H$ and by $P_{S}$ the projection operator on the space of all $n$-tensors over $H$ whose range is $H^{(n)}$. If $x_{1}, \ldots, x_{n}$ are in $H$ then we put $P_{S}\left(x_{1} \otimes x_{2} \otimes \cdots \otimes x_{n}\right)=x_{1} x_{2} \cdots x_{n}$ and $x^{n}=x \otimes \cdots \otimes x$ ( $n$ factors). An operator $A$ on a Hilbert space is of trace class if trace $\left[\left(A^{*} A\right)^{1 / 2}\right]$ converges on some orthonormal basis. As is known [6] the operators of trace class on a Hilbert space form a Banach space under the norm $\|A\|_{1}=\operatorname{trace}\left[\left(A^{*} A\right)^{1 / 2}\right]$.

Definition. A bounded linear operator $A$ from $H^{(n)}$ into $H^{(n)}$ is called completely symmetric if for any finite sets of vectors $x_{1}, \cdots, x_{n}$ and $y_{1}, \cdots, y_{n}$ in $H$ we have

$$
\left(A x_{1} x_{2} \cdots x_{n}, y_{1} \cdots y_{n}\right)=\left(A y_{1} x_{2} \cdots x_{n}, x_{1} y_{2} \cdots y_{n}\right) \text {. }
$$

REMARKs. (1) If $A$ is completely symmetric then it is symmetric. This follows from the fact that vectors of the form $x_{1} \cdots x_{n}$ span $H^{(n)}$.

(2) If $A$ is a Hilbert-Schmidt operator on $H^{(n)}$ i.e. trace $A^{*} A<\infty$ and if $w$ is the tensor in $H^{(n)} \otimes H^{(n)}$ which corresponds to $A$ via the relation $(A u, v)=$ $(w, u \otimes v)$ then $w$ may also.be regarded as an element of $H \otimes H \otimes \ldots \otimes H(2 n$ factors). $A$ is completely symmetric if and only if $w$ is in $H^{(2 n)}$. For if $A$ is completely symmetric then $\left(w,\left(x_{1} \ldots x_{n}\right) \otimes\left(x_{n+1} \ldots x_{2 n}\right)\right)$ is invariant under all permutations of the $x_{i}$, hence $(w, u \otimes v)=\left(w, P_{S}(u \otimes v)\right)$ for all $u, v \in H^{(n)}$ where $P_{S}$ is here the symmetry projection on the $2 n$-tensors over $H$. Thus $(A u, v)=\left(P_{S} w, u \otimes v\right)$. Since $P_{S} w$ is in $H^{(2 n)} \subset H^{(n)} \otimes H^{(n)}$ and since there is only one $w$ in in $H^{(n)} \otimes H^{(n)}$ for which $(A u, v)=(w, u \otimes v)$ it follows that $w=P_{s} w$. The converse is clear. Much of the following could be phrased in terms of the symmetric $2 n$-tensor $w$. However operator theoretic properties of $A$ play a dominant role.

THEOREM 3. If $A$ is a completely symmetric operator on $H^{(n)}$ of trace class such that for all $x, y$ in $H\left(A x^{n-1} y, x^{n-1} y\right) \geqq 0$ then the function $\|x\|_{A}=$ $\left(A x^{n}, x^{n}\right)^{1 / 2 n}$ is a measurable pseudo-norm on $H$. Furthermore $E\left[(\|\cdot\| \tilde{A})^{2 n}\right]=$ $c^{n} 1 \cdot 3 \cdots(2 n-1)$ trace $(A)$ where $E$ denotes expectation with respect to the canonical normal distribution on $H$ with variance parameter $c$.

LEMMA 3.1. If $H$ is finite dimensional then every completely symmetric operator on $H^{(n)}$ is a finite linear combination of projections of the form $P v=$ $x^{n}\left(v, x^{n}\right)$ where $x$ is a unit vector in $H$.

Proof. The symmetric $2 n$-tensor corresponding as in Remark (2) to the operator $A v=\sum a_{j} x_{j}^{n}\left(v, x_{j}^{n}\right)$ is $\sum a_{j} x_{j}^{2 n}$. Thus by Remark (2) it suffices to show that the vectors $x^{2 n} \operatorname{span} H^{(2 n)}$. One may prove easily by induction that the 
vectors $x^{m}$ span $H^{(m)}$. Assuming that $w$ is in $H^{(m)}$ and that $\left(w, x^{m}\right)=0$ for all $x$ the induction step consists in setting equal to zero the coefficient of $s$ in the expansion $0=\left(w,(s x+y)^{m}\right)=\left(w, y^{m}\right)+s m\left(w, x y^{m-1}\right)+\cdots$ thereby obtaining with the help of the induction assumption $\left(w, P_{S}(x \otimes u)\right)=0$ for all $u$ in $H^{(m-1)}$ and in particular $\left(w, x_{1} x_{2} \cdots x_{m}\right)=0$ for all $x_{j}$ in $H$ so that $w=0$.

The formula in the following corollary is due to Seidman and Friedrichs [3].

COROLlary 3.1. If $H$ is a finite dimensional Hilbert space and $A$ is a completely symmetric operator on $H^{(n)}$ and if $E$ is as in the theorem then $E\left[\left(A x^{n}, x^{n}\right)\right]$ $=c^{n} 1 \cdot 3 \cdots(2 n-1)$ trace $A$.

Proof. Writing $A v=\sum a_{j} x_{j}^{n}\left(v, x_{j}^{n}\right)$ where $x_{j}, j=1, \cdots, q$ are unit vectors in $H$ we have $E\left[\left(A x^{n}, x^{n}\right)\right]=\sum a_{j} E\left[\left(x, x_{j}\right)^{2 n}\right]=\sum a_{j}(2 \pi c)^{-1 / 2} \int_{-\infty}^{\infty} t^{2 n} \exp \left[-t^{2} / 2 c\right] d t$ $=c^{n} 1 \cdot 3 \cdots(2 n-1) \sum a_{j}=c^{n} 1 \cdot 3 \cdots(2 n-1)$ trace $A$.

LEMMA 3.2. Let $\mathscr{R}$ be a bounded (in operator norm) set of bounded linear operators on a (real or complex) Hilbert space $K$. If $A$ is an operator of trace class on $K$ then the map $B \rightarrow B A$ from $\mathscr{R}$ to the Banach space of trace class operators on $K$ is continuous in the strong operator topology on $\mathscr{R}$.

Proof. If $S=V C$ is the polar composition of $A$ with $C=\left(A^{*} A\right)^{1 / 2}$ then the orthogonal complement of the null space of $C$ is separable and has an orthonormal basis $x_{1}, x_{2}, \ldots$ such that $C x_{j}=\lambda_{j} x_{j} j=1,2, \ldots$ Then $\lambda_{j}>0$ and $\sum \lambda_{j}$ $=\operatorname{tr} C<\infty$. Let $\left\{y_{\alpha}\right\}$ be an orthonormal basis of the null space of $C$. If $\|B\| \leqq r$ for all $B$ in $\mathscr{R}$ and if $D=B-B_{0}$ then

$$
\begin{aligned}
\left\|B A-B_{0} A\right\|_{1} & =\operatorname{tr}\left[\left((D A)^{*} D A\right)^{1 / 2}\right] \\
& =\sum_{j}\left(\left(A^{*} D^{*} D A\right)^{1 / 2} x_{j}, x_{j}\right)+\Sigma_{\alpha}\left(\left(A^{*} D^{*} D A\right)^{1 / 2} y_{\alpha}, y_{\alpha}\right) \\
& \leqq \Sigma_{j}\left\|\left(A^{*} D^{*} D A\right)^{1 / 2} x_{j}\right\|+\Sigma_{\alpha}\left\|\left(A^{*} D^{*} D A\right)^{1 / 2} y_{\alpha}\right\| \\
& \leqq \Sigma_{j}\left(A^{*} D^{*} D A x_{j}, x_{j}\right)^{1 / 2}+\Sigma_{\alpha}\left(A^{*} D^{*} D A y_{\alpha}, y_{\alpha}\right)^{1 / 2} \\
& \leqq \sum_{j} \lambda_{j}\left\|D V x_{j}\right\| .
\end{aligned}
$$

If $N$ is so large that $2 r \sum_{j>N} \lambda_{j}<\varepsilon / 2$ and if $B$ is in the strong neighborhood of $B_{0}$ defined by $\|A\|\left\|\left(B-B_{0}\right) V x_{j}\right\|<\varepsilon / 2 N, j=1, \ldots, N$, then $\left\|B A-B_{0} A\right\|_{1}<\varepsilon$ which establishes the lemma.

COROLlaRy 3.2. If $A$ is a trace class operator on a (real or complex) Hilbert space $K$ and if $P_{\alpha}$ is a net of projections which converge strongly to the identity then $P_{\alpha} A P_{\alpha}$ converges in trace class norm to $A$.

Proof. $\quad\left\|P_{\alpha} A P_{\alpha}-A\right\|_{1} \leqq\left\|P_{\alpha} A P_{\alpha}-A P_{\alpha}\right\|_{1}+\left\|A P_{\alpha}-A\right\|_{1} \leqq\left\|P_{\alpha} A-A\right\|_{1}$ $+\left\|P_{\alpha} A^{*}-A^{*}\right\|_{1}$. Apply Lemma 3.2.

LEMMA 3.3. If $A$ is a completely symmetric bounded operator on $H^{(n)}$ then 
$\left(A x^{n}, x^{n}\right)$ is the $2 n t h$ power of a pseudo-norm on $H$ if and only if $\left(A x^{n-1} y, x^{n-1} y\right)$ $\geqq 0$ for all $x, y$ in $H$.

Proof. If $\|x\|_{A}=\left(A x^{n}, x^{n}\right)^{1 / 2 n}$ is a pseudo-norm then $\|x+t y\|_{A}$ is a convex function of $t$ for all $x$ and $y$ hence so is $\|x+t y\|_{A}^{2 n}$. The second derivative of $\left(A(x+t y)^{n},(x+t y)^{n}\right)$ with respect to $t$ at $t=0$ is therefore non-negative i.e. $2 n(2 n-1)\left(A x^{n-1} y, x^{n-1} y\right) \geqq 0$. Conversely if $\left(A x^{n-1} y, x^{n-1} y\right) \geqq 0$ for all $x, y$ then it follows easily that the second derivative of $\left(A(x+t y)^{n},(x+t y)^{n}\right)$ is nonnegative for all $t$, that this is therefore a convex function of $t$, that the set of $x$ for which $\left(A x^{n}, x^{n}\right) \leqq 1$ is a convex set and that the positive homogeneous function $\left(A x^{n}, x^{n}\right)^{1 / 2 n}$ (which is defined because $\left(A x^{n}, x^{n}\right) \geqq 0$ for all $x$ ) has a convex unit sphere and is therefore a pseudo-norm.

Proof of theorem. For each finite dimensional projection $Q$ on $H$ we denote by $Q^{(n)}$ the projection $Q \otimes Q \otimes \cdots \otimes Q$ on $H^{(n)}$. As $Q$ approaches the identity through the directed set $\mathscr{F}$ of finite dimensional projections on $H, Q^{(n)}$ converges strongly to the identity on $H^{(n)}$. If $A$ is a trace class operator on $H^{(n)}$ then $Q^{(n)} A Q^{(n)}$ converges to $A$ in trace class norm by Corollary 3.2. Thus if $A$ satisfies the hypotheses of the theorem and if $\varepsilon>0$ then there exists a finite dimensional projection $P_{0}$ on $H$ such that $\left\|A-P_{0}^{(n)} A P_{0}^{(n)}\right\|_{1}<\varepsilon^{2 n+1}$. Let $P$ be a finite dimensional projection orthogonal to $P_{0}$ then $P^{(n)} P_{0}^{(n)}=0$ and $\left\|P^{(n)} A P^{(n)}\right\|_{1}=\left\|P^{n}\left(A-P_{0}^{(n)} A P_{0}^{(n)}\right) P^{(n)}\right\|_{1}<\varepsilon^{2 n+1}$. By Lemma $3.3\|x\|_{A}=\left(A x^{n}, x^{n}\right)^{1 / 2 n}$ is a pseudo-norm. Now $P^{(n)} A P^{(n)}$ is a completely symmetric operator on $H^{(n)}$ and for the purpose of evaluating $E\left[\left(P^{(n)} A P^{(n)} x^{n}, x^{n}\right)\right]$ we may regard $P^{(n)} A P^{(n)}$ as an operator on $(P H)^{(n)}$ and apply Corollary 3.1. Thus we have

$$
E\left[\|P x\|_{A}^{2 n}\right]=c^{n} 1 \cdot 3 \cdots(2 n-1) \operatorname{tr}\left(P^{(n)} A P^{(n)}\right)<c^{n} 1 \cdot 3 \cdots(2 n-1) \varepsilon^{2 n+1} .
$$

Hence Prob $\left(\|P x\|_{A}>\varepsilon\right)<c^{n} 1 \cdot 3 \cdots(2 n-1) \varepsilon$. Hence $\|x\|_{A}$ is a measurable pseudo-norm. To derive the final formula of the theorem observe that if $P$ and $Q$ are in $\mathscr{F}$ then $\|P x\|_{A}-\|Q x\|_{A} \mid \leqq\|(P-Q) x\|_{A}$ and that $E\left[\|(P-Q) x\|_{A}^{2 n}\right]=c^{n} 1 \cdot 3 \cdot 5 \cdots(2 n-1)$ trace $\left[\left((P-Q)^{(n)}\right)^{2} A\right]$ which goes to zero as $P$ and $Q$ approach the identity since $(P-Q)^{(n)} \rightarrow 0$ strongly and Lemma 3.2 applies. Thus $\|P x\|_{A}$ converges to $\|\cdot\| \tilde{A}$ not only in probability but also in $L^{2 n}$. Hence

$$
\begin{aligned}
& E\left[\left(\|\cdot\|_{A}\right)^{2 n}\right]=\lim _{P \rightarrow I} E\left[\|P x\|_{A}^{2 n}\right] \\
& \quad=\lim _{P \rightarrow I} c^{n} 1 \cdot 3 \cdots(2 n-1) \cdot \operatorname{trace} P^{(n)} A P^{(n)}=c^{n} 1 \cdot 3 \cdots(2 n-1) \text { trace } A .
\end{aligned}
$$

EXAMPLES. (1) Let $x_{1}, x_{2}, \cdots$ be an orthonormal basis of a real Hilbert space $H$. Let $a_{j} \geqq 0$ be a sequence of real numbers such that $\sum_{j} a_{j}<\infty$. Let $A: H^{(n)}$ $\rightarrow H^{(n)}$ be defined by $A=\sum a_{j} P_{j}$ where $P_{j}$ is the one dimensional projection $P_{j} v=x_{j}^{n}\left(v, x_{j}^{n}\right)$. If $x=\sum_{j} t_{j} x_{j}$ then $\|x\|_{A}=\left(\sum_{j} a_{j} t_{j}^{2 n}\right)^{1 / 2 n}$ is a measurable pseudonorm. 
(2) If $A$ is a non-negative trace class operator on $H$ then $\|x\|_{A}=(A x, x)^{1 / 2}$ is the typical measurable pseudo-norm used in [5].

4. General properties of measurable pseudo-norms. In order to establish that a given pseudo-norm is measurable it is convenient to have some convergence theorems for measurable pseudo-norms. The following theorem and its corollaries will suffice us for the examples of the next section. A limit indicated by $P \rightarrow I$ means the limit as $P \rightarrow I$ through the directed set $\mathscr{F}$.

THEOREM 4. Let $\|x\|_{n}$ be a nondecreasing sequence of pseudo-norms on $H$ which converge for each $x$ in $H$. Let $\|x\|_{0}=\lim _{n \rightarrow \infty}\|x\|_{n}$. Assume further the following

(1) lim in $\operatorname{Prob}_{P \rightarrow I}\|P x\|_{n}=h_{n}$ exists for each $n$.

(2) $h=\lim _{\text {in }}$ Prob $_{n \rightarrow \infty} h_{n}$ exists.

(3) $\operatorname{Prob}(h \leqq \varepsilon)>0$ for every $\varepsilon>0$.

Then $\|x\|_{0}$ is a measurable pseudo-norm and $\|x\|_{0}=h$.

LEMMA 4.1. Let $S$ be a compact convex set in $E_{n}$. Let $p_{1}, p_{2}, p_{3}$ be three parallel hyperplanes in $E_{n}$ such that $p_{2}$ lies between $p_{1}$, and $p_{3}$ (i.e. $p_{2}$ is in the convex closure of $p_{1}$ and $\left.p_{3}\right)$. Let $S_{i}=S \cap p_{i}, i=1,2,3$ and let $m$ denote $n-1$ dimensional Lebesgue measure. Then $m\left(S_{2}\right) \geqq \min \left\{m\left(S_{1}\right), m\left(S_{3}\right)\right\}$.

Proof. This is a simple consequence of the Brunn-Minkowski theorem. (See $[1, \S 41]$.)

LEMMA 4.2. Let $F$ be a subspace of a finite dimensional real Hilbert space $K$. Denote by $\mu$ and $\mu^{\prime}$ Gauss measure ${ }^{2}$ ) in $K$ and $F$ respectively. Let $B$ be a convex centrally symmetric set in $K$ and let $C$ be a convex cylinder in $K$ of the form $C=D \times F$ where $D$ is a convex set in $F^{\perp}$. If $B$ is contained in $C$ then $\mu(B) \leqq$ $\mu(C) \mu^{\prime}(F \cap B)$. In particular if $C=K$ then $\mu(B) \leqq \mu^{\prime}(F \cap B)$.

Proof. Let $x \neq 0$ be in $F^{\perp}$ and denote by $P$ the orthogonal projection on $K$ with range $F$. Let $r>0$ and let $C_{r}$ denote the circular cylinder $\{x:\|P x\| \leqq r\}$. If $k$ is the dimension of $F$ then we denote by $m, k$-dimensional Lebesgue measure. In the $k+1$ dimensional subspace $G$ spanned by $x$ and $F$ the previous lemma may be applied to the three hyperplanes $-x+F, F$ and $x+F$ and to the convex set $S_{r}=G \cap B \cap C_{r}$ to obtain

$$
m\left(F \cap S_{r}\right) \geqq \min \left\{m\left((-x+F) \cap S_{r}\right), m\left((x+F) \cap S_{r}\right)\right\} .
$$

(The possible lack of compactness of $S_{r}$ clearly does not affect the application of Lemma 4.1). Since $S_{r}$ is centrally symmetric we have $m\left(F \cap S_{r}\right) \geqq m\left((x+F) \cap S_{r}\right)$. Hence

(2) Gauss measure on a $k$-dimensional real space refers to the probability measure $(2 \pi)^{-k / 2} \exp \left(-\|x\|^{2 / 2}\right) d x$ where $d x$ is Lebesgue measure. 


$$
\begin{aligned}
\mu^{\prime}((x+F) \cap B) & =\mu^{\prime}((x+F) \cap(G \cap B)) \\
& =(2 \pi c)^{-k / 2} \int_{0}^{\infty} e^{-r^{2} / 2 c} d_{r} m\left((x+F) \cap S_{r}\right) \\
& =(2 \pi c)^{-k / 2} \int_{0}^{\infty} m\left((x+F) \cap S_{r}\right) e^{-r^{2} / 2 c}(r / c) d r \\
& \leqq(2 \pi c)^{-k / 2} \int_{0}^{\infty} m\left(F \cap S_{r}\right) e^{-r^{2} / 2 c}(r / c) d r \\
& \leqq \mu^{\prime}(F \cap B) .
\end{aligned}
$$

Now let $v$ be Gauss measure in $F^{\perp}$. Then $\mu$ is the product of $\mu^{\prime}$ and $v$. Now the projection of $B$ on $F^{+}$is contained in the projection of $C$ on $F^{+}$which is is exactly $C \cap F^{+}=D$. Hence

$$
\begin{aligned}
\mu(B) & =\int_{F^{+}} \mu^{\prime}((x+F) \cap B) d v(x) \\
& =\int_{D} \mu^{\prime}((x+F) \cap B) d v(x) \\
& \leqq \int_{D} \mu^{\prime}(F \cap B) d v(x) \\
& \leqq \mu^{\prime}(F \cap B) v(D)=\mu^{\prime}(F \cap B) \mu(C) .
\end{aligned}
$$

COROLlaRY 4.3. If $\|x\|_{1}$ is a pseudo-norm on $H$ and $P$ and $Q$ are in $\mathscr{F}$ and $P \leqq Q$ then for all $a>0 \operatorname{Prob}\left(\|P x\|_{1}>a\right) \leqq \operatorname{Prob}\left(\|Q x\|_{1}>a\right)$.

Proof. Take $K=Q H, F=P H, B=\left\{x \in Q H:\|Q x\|_{1} \leqq a\right\}$. Then the preceding lemma yields $\operatorname{Prob}\left(\|Q x\|_{1} \leqq a\right)=\mu(B) \leqq \mu^{\prime}(F \cap B)=\operatorname{Prob}\left(\|P x\|_{1} \leqq a\right)$.

Proof of theorem. It is clear that $\|x\|_{0}$ is a pseudo-norm. It suffices to prove that if $\varepsilon>0$ is a continuity point of the distribution function of $h$ and also of the distribution functions of all the $h_{n}$ and if $\delta$ is given with $0<\delta<1$ then there exists a finite dimensional projection $P_{0}$ such that Prob $\left(\|P x\|_{0}>\varepsilon\right) \leqq \delta$ whenever $P$ is in $\mathscr{F}$ and $P \perp P_{0}$.

Let $\eta=\delta /(3-\delta)$ and let $a=\operatorname{Prob}(h \leqq \varepsilon)$. Now Prob $\left(h_{m} \leqq \varepsilon\right)$ converges to $\operatorname{Prob}(h \leqq \varepsilon)=a$ as $m \rightarrow \infty$ and also $\operatorname{Prob}\left(\|Q x\|_{m} \leqq \varepsilon\right)$ converges to $\operatorname{Prob}\left(h_{m} \leqq \varepsilon\right)$ as $Q$ approaches $I$ through $\mathscr{F}$ for each $m$. Hence there exists an $N$ and for each $m \geqq N$ a finite dimensional projection $Q_{m}$ such that $\left|\operatorname{Prob}\left(\|Q x\|_{m} \leqq \varepsilon\right)-a\right| \leqq \eta a$ for $m \geqq N$ and $Q \geqq Q_{m}$. Let $X(t)$ be the characteristic function of $(-\infty, \varepsilon]$. It is not hard to show that $X\left(\|Q x\|_{N}\right)$ converges to $X\left(h_{N}\right)$ in $L^{2}$ as $Q \rightarrow I$ through $\mathscr{F}$. Hence there exists $P_{0}$ in $\mathscr{F}$ which we may and shall take $\geqq Q_{N}$ such that $\left\|X\left(\|Q x\|_{N}\right)-X\left(h_{N}\right)\right\|_{L}^{2} \leqq \eta a / 2$ for all $Q \geqq P_{0}(Q \in \mathscr{F})$.

Now let $P$ be in $\mathscr{F}$ and let $P \perp P_{0}$. Let $Q$ be in $\mathscr{F}$ and suppose $Q \geqq P_{0}+P$ 
while $m \geqq N$. In the space $K=Q H$ we consider the following centrally symmetric convex sets

$$
\begin{aligned}
& B_{1}=\left\{x \in Q H:\left\|P_{0} x\right\|_{N} \leqq \varepsilon\right\}, \\
& B_{2}=\left\{x \in Q H:\|x\|_{m} \leqq \varepsilon\right\}, \\
& B_{3}=B_{1} \cap B_{2}, \\
& B_{4}=\left\{x \in Q H:\|x\|_{N} \leqq \varepsilon\right\} .
\end{aligned}
$$

If $\mu$ denotes Gauss measure in $K$ then $\mu\left(B_{1}\right)=\operatorname{Prob}\left(\left\|P_{0} x\right\|_{N} \leqq \varepsilon\right)$ and similarly for other probabilities. Let $F$ be the subspace $\left(Q-P_{0}\right) H$ and let $\mu^{\prime}$ be Gauss measure in $F . B_{1}$ is a cylinder parallel to $F$. Since $B_{3} \subset B_{1}$ and $B_{3}$ is centrally symmetric we have from Lemma $4.2 \mu\left(B_{3}\right) \leqq \mu\left(B_{1}\right) \mu^{\prime}\left(B_{3} \cap F\right)$. As $F \subset B_{1}$ it follows that $B_{3} \cap F=B_{2} \cap F \quad$ so that $\mu^{\prime}\left(B_{3} \cap F\right)=\mu^{\prime}\left(B_{2} \cap F\right)=$ $\operatorname{Prob}\left(\left\|\left(Q-P_{0}\right) x\right\|_{m} \leqq \varepsilon\right)$. Hence

$$
\mu\left(B_{3}\right) \leqq \operatorname{Prob}\left(\left\|P_{0} x\right\|_{N} \leqq \varepsilon\right) \operatorname{Prob}\left(\left\|\left(Q-P_{0}\right) x\right\|_{m} \leqq \varepsilon\right) .
$$

Furthermore $\|x\|_{m} \geqq\|x\|_{N}$ so that $B_{2} \subset B_{4}$. Thus $\mu\left(B_{2}-B_{1}\right) \leqq \mu\left(B_{4}-B_{1}\right)$ $\leqq \mu\left(\left(B_{4}-B_{1}\right) \cup\left(B_{1}-B_{4}\right)\right)=E\left[\left(X\left(\left\|P_{0} x\right\|\right)-X\left(\|Q x\|_{N}\right)\right)^{2}\right] \leqq \eta a$. Consequently as $\operatorname{Prob}\left(\|Q x\|_{m} \leqq \varepsilon\right)=\mu\left(B_{2}\right)=\mu\left(B_{3}\right)+\mu\left(B_{2}-B_{1}\right)$ we have

$$
\operatorname{Prob}\left(\|Q x\|_{m} \leqq \varepsilon\right) \leqq \operatorname{Prob}\left(\left\|P_{0} x\right\|_{N} \leqq \varepsilon\right) \operatorname{Prob}\left(\left\|\left(Q-P_{0}\right) x\right\|_{m} \leqq \varepsilon\right)+\eta a
$$

for all $Q \geqq P_{0}+P$ and all $m \geqq N$.

Now $P H$ is a subspace of $\left(Q-P_{0}\right) H$ so that from Corollary 4.3 it follows that $\operatorname{Prob}\left(\left\|\left(Q-P_{0}\right) x\right\|_{m} \leqq \varepsilon\right) \leqq \operatorname{Prob}\left(\|P x\|_{m} \leqq \varepsilon\right)$. Furthermore if $Q \geqq Q_{m}$ then from the definition of $Q_{m}$ we have $\operatorname{Pr}\left(\|Q x\|_{m} \leqq \varepsilon\right) \geqq(1-\eta) a$ and as $P_{0} \geqq Q_{N}$ we have $\operatorname{Pr}\left(\left\|P_{0} x\right\|_{N} \leqq \varepsilon\right) \leqq(1+\eta) a$. Making these substitutions in (1) we obtain when $Q \geqq Q_{m} \vee\left(P_{0}+P\right)$

$$
(1-\eta) a \leqq(1+\eta) a \operatorname{Prob}\left(\|P x\|_{m} \leqq \varepsilon\right)+\eta a .
$$

Upon cancelling $a$ and collecting terms we obtain

$$
\operatorname{Prob}\left(\|P x\|_{m} \leqq \varepsilon\right) \geqq(1-2 \eta) /(1+\eta)=1-\delta
$$

for $P \perp P_{0}$ and $m \geqq N$. In the finite dimensional space $P H$ the pseudo-norm $\|x\|_{0}$ has a continuous distribution function with respect to Gauss measure in $P H$. Since $\|P x\|_{m}$ converges to $\|P x\|_{0}$ everywhere in $P H$ it converges in probability and consequently $\operatorname{Prob}\left(\|P x\|_{0} \leqq \varepsilon\right)=\lim _{m \rightarrow \infty} \operatorname{Pr}\left(\|P x\|_{m} \leqq \varepsilon\right) \geqq 1-\delta$ whenever $P \perp P_{0}$. This proves that $\|x\|_{0}$ is a measurable pseudo-norm.

To conclude the proof of the theorem let $g=\|x\|_{0}$. Observe that if $R \geqq P_{0}$ then $\operatorname{Prob}\left(\left\|\left(R-P_{0}\right) x\right\|_{m}>\varepsilon\right) \leqq \delta$ for $m \geqq N$ and also for $m=0$. Hence

$$
\begin{aligned}
\operatorname{Prob}\left(\mid h_{m}-\left\|P_{0} x\right\|_{m}>2 \varepsilon\right) & \leqq \lim _{R \rightarrow I} \operatorname{Prob}\left(\|R x\|_{m}-\left\|P_{0} x\right\|_{m}>\varepsilon\right) \\
& \leqq \lim _{R \rightarrow I} \operatorname{Prob}\left(\left\|\left(R-P_{0}\right) x\right\|_{m}>\varepsilon\right) \leqq \delta .
\end{aligned}
$$


Similarly for $\|x\|_{0}$. Hence in virtue of the inequality

$|g-h| \leqq\left|g-\left\|P_{0} x\right\|_{0}\right|+\left|\left\|P_{0} x\right\|_{0}-\left\|P_{0} x\right\|_{m}\right|+\left|\left\|P_{0} x\right\|_{m}-h_{m}\right|+\left|h_{m}-h\right|$

it follows that $\operatorname{Prob}(|g-h|>6 \varepsilon) \leqq 2 \delta+\operatorname{Prob}\left(\left|\left\|P_{0} x\right\|_{0}-\left\|P_{0} x\right\|_{m}\right|>\varepsilon\right)+$ $\operatorname{Prob}\left(\left|h_{m}-h\right|>\varepsilon\right)$. The last two terms go to zero as $m \rightarrow \infty$ which establishes that $g=h$.

COROLLARY 4.4. Let $\|x\|_{n}$ be a nondecreasing sequence of measurable pseudonorms. Suppose that $h=\lim _{\text {in }}$ prob $_{n_{n \rightarrow \infty}}\|x\|_{n}^{\tilde{n}}$ exists and has the property that $\operatorname{Prob}(h \leqq \varepsilon)>0$ for all $\varepsilon>0$. Then $\|x\|_{0}=\lim _{n \rightarrow \infty}\|x\|_{n}$ is a measurable pseudo-norm.

Proof. The corollary will follow immediately from the theorem as soon as we show that $\lim _{n \rightarrow \infty}\|x\|_{n}$ exists for each $x$ in $H$. If $x_{0}$ is such that $\lim _{n \rightarrow \infty}\left\|x_{0}\right\|_{n}$ $=\infty$ then also $\|x\|_{n}$ diverges to infinity on the one dimensional subspace spanned by $x_{0}$ except at $x=0$. Hence if $P$ is the one dimensional projection onto the span of $x_{0}$ then for any real number $a$ we have $\operatorname{Prob}\left(\|P x\|_{n} \leqq a\right) \rightarrow 0$ as $n \rightarrow \infty$. If $Q$ is a finite dimensional projection dominating $P$ then by Corollary 4.3 we have $\operatorname{Prob}\left(\|Q x\|_{n} \leqq a\right) \leqq \operatorname{Prob}(\|P x\| \leqq a)$ and letting $Q \rightarrow I$ we have

$$
\operatorname{Prob}\left(\|x\|_{n} \leqq a\right) \leqq \operatorname{Prob}\left(\|P x\|_{n} \leqq a\right)
$$

whenever $a$ is a continuity point of the distribution function of $\|x\|_{n}$. Thus if $a$ is a continuity point of the distribution function of all the random variables $\|x\|_{n}$ then $\operatorname{Prob}\left(\|x\|_{n} \leqq a\right) \rightarrow 0$ as $n \rightarrow \infty$ which contradicts the assumption that the sequence $\|x\|_{n}$ converges in probability.

COROLlary 4.5. Let $\|x\|_{0}$ be a pseudo-norm on $H$. Then $\|x\|_{0}$ is a measurable pseudo-norm if and only if $h=\lim$ in prob $_{P \rightarrow I}\|P x\|_{0}$ exists and has the property that $\operatorname{Prob}(h \leqq \varepsilon)>0$ for every $\varepsilon>0$.

Proof. Half of the corollary is the assertion of Theorem 1. The other half follows from Theorem 4 upon considering the sequence $\|x\|_{n}=\|x\|_{0}$ which clearly satisfies the conditions of the theorem.

THEOREM 5. If $A$ is a bounded linear operator on a real Hilbert space $H$ and $\|x\|_{1}$ is a measurable pseudo-norm on $H$ then the pseudo-norm $\|x\|_{2}$ $=\|A x\|_{1}$ is measurable and for every $\varepsilon>0$ Prob $\left(\|x\|_{2}>\|A\| \varepsilon\right)$ $\leqq \operatorname{Prob}(\|x\| \tilde{1}>\varepsilon)$. Furthermore $\|x\|_{2}=\lim$ in prob. $P \rightarrow I\|P A x\|_{1}$.

LEMMA 5.1. Let $T$ be a convex centrally symmetric set in $E_{n}$. If $B$ is a symmetric linear transformation on $E_{n}$ such that $\|B x\| \geqq\|x\|$ for all $x$ then $m\left(B T \cap S_{r}\right) \geqq m\left(T \cap S_{r}\right)$ for all $r>0$ where $S_{r}$ is the sphere of radius $r$ centered at the origin and $m$ denotes Lebesgue measure.

Proof. If the lemma holds for $B_{1}$ and $B_{2}$ and all centrally symmetric convex 
sets $T$ then it also holds for $B=B_{1} B_{2}$ for $m\left((B T) \cap S_{r}\right)=m\left(\left(B_{1} B_{2} T\right) \cap S_{r}\right)$ $\geqq m\left(B_{2} T \cap S_{r}\right) \geqq m\left(T \cap S_{r}\right)$. It suffices therefore to prove the lemma for a transformation $B$ whose matrix on an orthonormal basis $x_{1}, \cdots, x_{n}$ is diagonal with diagonal entries $b, 1,1, \cdots, 1$ where $b \geqq 1$.

Let $l_{h}$ be the hyperplane whose equation is $\left(x, x_{1}\right)=h$, and let $m^{\prime}$ denote $n-1$ dimensional Lebesgue measure. We note that $m\left(T \cap S_{r}\right)=\int_{-r}^{r} m^{\prime}\left(T \cap S_{r} \cap l_{h}\right) d h$. Let $T_{h}=T \cap S_{r} \cap l_{h}$. Since $T$ and $S_{r}$ are centrally symmetric it follows that $T_{-h}$ is the reflection of $T_{h}$ in the origin. Hence $m^{\prime}\left(T_{h}\right)=m^{\prime}\left(T_{-h}\right)$. Let $D_{h}$ be the convex set generated by $T_{h}$ and $T_{-h}$. Then $D_{h} \subset T \cap S_{r}$ and $D_{h} \cap l_{h}=T_{h}$ while $D_{h} \cap l_{-h}=T_{-h}$. Hence by Lemma $4.1 m^{\prime}\left(D_{h} \cap l_{h / b}\right) \geqq m^{\prime}\left(T_{h}\right)$. Since $B l_{h / b}$ $=l_{h}$ we have $S_{r} \cap B T \cap l_{h}=S_{r} \cap B\left(T \cap l_{h / b}\right) \supset S_{r} \cap B\left(D_{h} \cap l_{h / b}\right)$. Furthermore it is easy to see that $B\left(D_{h} \cap l_{h / b}\right) \subset S_{r}$ since $D_{h}$ is contained in the convex set (a cylinder) generated by $S_{r} \cap l_{h}$ and $S_{r} \cap l_{-h}$. Hence $S_{r} \cap B T \cap l_{h} \supset$ $B\left(D_{h} \cap l_{h / b}\right)$ and $m^{\prime}\left(S_{r} \cap B T \cap l_{h}\right) \geqq m^{\prime}\left(B\left(D_{h} \cap l_{h / b}\right)\right)=m^{\prime}\left(D_{h} \cap l_{h / b}\right) \geqq m^{\prime}\left(T_{h}\right)$. Thus $m\left(B T \cap S_{r}\right)=\int_{-r}^{r} m^{\prime}\left(S_{r} \cap B T \cap l_{h}\right) d h \geqq \int_{-r}^{r} m^{\prime}\left(T_{h}\right) d h=m\left(T \cap S_{r}\right)$.

LEMMA 5.2. Under the hypotheses of the preceding lemma we have $\mu(T)$ $\leqq \mu(B T)$ where $\mu$ denotes Gauss measure in $E_{n}$.

Proof.

$$
\begin{aligned}
(2 \pi c)^{n / 2} \mu(T) & =\int_{0}^{\infty} e^{-r^{2} / 2 c} d_{r} m\left(T \cap S_{r}\right)=\int_{0}^{\infty} m\left(T \cap S_{r}\right) e^{-r^{2} / 2 c} r / c d r \\
& \leqq \int_{0}^{\infty} m\left(B T \cap S_{r}\right) e^{-r^{2} / 2 c} r / c d r=(2 \pi c)^{n / 2} \mu(B T)
\end{aligned}
$$

Proof of theorem. We shall first establish the following inequality for any finite dimensional projection $P$ and $\varepsilon>0$.

$$
\operatorname{Prob}\left(\|P A x\|_{1}>\|A\| \varepsilon\right) \leqq \operatorname{Prob}\left(\|P x\|_{1}>\varepsilon\right) .
$$

By dividing $A$ by $\|A\|$ we may assume $\|A\|=1$. Let $K$ be the orthogonal complement of the null space of $P A .\|P A x\|_{1}$ is a tame function based on $K$. Let $R=\left\{x \in K:\|P A x\|_{1} \leqq \varepsilon\right\}$ and let $T=\left\{x \in P H:\|x\|_{1} \leqq \varepsilon\right\}$. If $\mu$ and $\mu^{\prime}$ denote Gauss measure in $P H$ and $K$ respectively then (2) is equivalent to $\mu^{\prime}(R) \geqq \mu(T)$. Now let $F$ be the range of $P A$. Then $P A$ regarded as a transformation from $K$ to $F$ is nonsingular and we may write its polar composition $P A=C V$ where $V$ is an orthogonal transformation from $K$ onto $F$ and $C$ is a strictly positive transformation on $F$ with $\|C\| \leqq 1$. Now $P A(R)$ is the intersection of $T$ with $F$. Hence if $\mu^{\prime \prime}$ denotes Gauss measure in $F$ then by Lemmas 4.2 and $5.2 \mu(T) \leqq \mu^{\prime \prime}(T \cap F)$ $=\mu^{\prime \prime}(C V(R)) \leqq \mu^{\prime \prime}\left(C^{-1} C V(R)\right)=\mu^{\prime \prime}(V(R))=\mu^{\prime}(R)$ since $C^{-1}$ satisfies the hypothesis of Lemma 5.2. This establishes (2).

To show that $\|x\|_{2}$ is measurable let $P_{0}$ be a finite dimensional projection such that $\operatorname{Prob}\left(\|P x\|_{1}>\varepsilon\right)<\varepsilon$ whenever $P \perp P_{0}$ and $P$ is in $\mathscr{F}$. Let $Q_{0}$ be the (finite 
dimensional) projection onto the orthocomplement of the null space of $P_{0} A$. If $Q$ is in $\mathscr{F}$ and $Q$ is orthogonal to $Q_{0}$ then the projection $P$ onto the range of $A Q$ is orthogonal to $P_{0}$. Furthermore $P A Q=A Q$. Thus in virtue of Corollary 4.3 and inequality (2) we have $\operatorname{Prob}\left(\|Q x\|_{2}>\|A\| \varepsilon\right)=\operatorname{Prob}\left(\|P A Q x\|_{1}>\|A\| \varepsilon\right)$ $\leqq \operatorname{Prob}\left(\|P A x\|_{1}>\|A\| \varepsilon\right) \leqq \operatorname{Prob}\left(\|P x\|_{1}>\varepsilon\right)<\varepsilon$. Hence $\|x\|_{2}$ is measurable.

Now let $P_{n}^{\prime}$ be an increasing sequence of projections in $\mathscr{F}$ chosen as in the proof of Theorem 1 so that $\left\|P_{n}^{\prime} x\right\|_{1}$ converges to $\|x\| \tilde{1}$ in probability and let $Q_{n}$ be chosen similarly for $\|x\|_{2}$. Let $P_{n}^{\prime \prime}$ be the projection onto the range of $A Q_{n}$ and let $P_{n}=P_{n}^{\prime} \vee P_{n}^{\prime \prime}$. Then $\left\|P_{n} x\right\|_{1}$ also converges to $\|x\|_{1}$ in probability. Furthermore as $P_{n} A Q_{n}=A Q_{n}$ we have

$$
\begin{aligned}
\operatorname{Prob}\left(\left\|Q_{n} x\right\|_{2}\right. & >\|A\| \varepsilon) \\
& =\operatorname{Prob}\left(\left\|P_{n} A Q_{n} x\right\|_{1}>\|A\| \varepsilon\right) \leqq \operatorname{Prob}\left(\left\|P_{n} A x\right\|_{1}>\|A\| \varepsilon\right) \\
& \leqq \operatorname{Prob}\left(\left\|P_{n} x\right\|_{1}>\varepsilon\right.
\end{aligned}
$$

by a repeated application of Corollary 4.3 and inequality (2). Passing to the limit on $n$ we get the second assertion of the theorem for those $\varepsilon$ which are continuity points of the distribution functions of $\|x\| \tilde{1}$ and $\|A\|^{-1}\|x\|_{2}$ and hence for all $\varepsilon$.

Finally to show that the limit in probability of the net $\|P A x\|_{1}$ exists and equals the limit $h$ of the net $\|A Q x\|_{1}, P$ and $Q$ in $\mathscr{F}$ we observe that

$$
\begin{aligned}
& \operatorname{Prob}\left(\left|\|P A x\|_{1}-h\right|>3 \varepsilon\right) \\
& \quad \leqq \operatorname{Prob}\left(\left|\|P A x\|_{1}-\|A Q x\|_{1}\right|>2 \varepsilon\right)+\operatorname{Prob}\left(\left|\|A Q x\|_{1}-h\right|>\varepsilon\right) .
\end{aligned}
$$

Furthermore

$\left|\left\|P A_{x}\right\|_{1}-\|A Q x\|_{1}\right| \leqq\|(P A-A Q) x\|_{1} \leqq\|P A(I-Q) x\|_{1}+\|(I-P) A Q x\|_{1}$ so that

$$
\begin{aligned}
\operatorname{Prob} & \left(\left|\|P A x\|_{1}-\|A Q x\|_{1}\right|>2 \varepsilon\right) \\
& \leqq \operatorname{Prob}\left(\|P A(I-Q) x\|_{1}>\varepsilon\right)+\operatorname{Prob}\left(\|(I-P) A Q x\|_{1}>\varepsilon\right) \\
& \leqq \operatorname{Prob}(\|P A(I-Q) x\|>\varepsilon)+\operatorname{Prob}(\|(I-P) x\| \tilde{1}>\varepsilon /\|A\|)
\end{aligned}
$$

where in the last step we have made use of the fact already proven that $\left\|\left(I^{\prime}-P\right) x\right\|_{1}$ is a measurable pseudo-norm and $\|A Q\| \leqq\|A\|$, Now any measurable pseudonorm $\|x\|_{3}$ has the following property: if $\varepsilon>0$ and $\delta>0$ and $P_{0}$ is in $\mathscr{F}$ and is such that $\operatorname{Prob}\left(\|R x\|_{3}>\delta\right) \leqq \varepsilon$ whenever $R$ is in $\mathscr{F}$ and $R \perp P_{0}$ then for any $P$ in $\mathscr{F}$ with $P \geqq P_{0}$ we have

$$
\operatorname{Prob}\left(\|(I-P) x\|_{3}>2 \delta\right) \leqq \varepsilon .
$$

This follows easily from the relations

$$
\|(I-P) x\|_{3}=\lim \text { in } \operatorname{prob}_{Q \in \mathscr{F}}\|(I-P) Q x\|_{3}=\lim \text { in } \operatorname{prob}_{Q \in \mathscr{F}}\|(Q-P) x\|_{3}
$$


since $Q \geqq P$ implies $Q-P \perp P_{0}$. Therefore there exists $P_{0}$ in $\mathscr{F}$ such that $\operatorname{Prob}(\|(I-P) x\| \tilde{1}>\varepsilon /\|A\|)<\varepsilon$ whenever $P \geqq P_{0}$. Thus for $P \geqq P_{0}$ and $P$ in $\mathscr{F}$ we have

$$
\begin{aligned}
\operatorname{Prob}\left(\left|\|P A x\|_{1}-h\right|>3 \varepsilon\right) \leqq \varepsilon & +\operatorname{Prob}\left(\|P A(I-Q) x\|_{1}>\varepsilon\right) \\
& +\operatorname{Prob}\left(\left|\|A Q x\|_{1}-h\right|>\varepsilon\right) .
\end{aligned}
$$

Again since $\|P A x\|_{1}$ is a measurable pseudo-norm the second term on the right of the last inequality goes to zero as $Q \rightarrow I$ through $\mathscr{F}$ while the third does also by the definition of $h$. This concludes the proof of the theorem.

COROLlaRY 5.1. If $A_{\alpha}$ is a uniformly bounded net of bounded linear operators on $H$ for which $A_{\alpha}^{*}$ converges strongly to $A^{*}$ and if $\|x\|_{1}$ is a measurable pseudonorm on $H$ then $\left\|A_{\alpha} x\right\|_{1}$ converges in probability to $\|A x\| \tilde{1}$.

Proof. Let $M=\sup _{\alpha}\left\|A_{\alpha}\right\|$. Let $P_{0}$ in $\mathscr{F}$ be such that $P \perp P_{0}$ and $P$ in $\mathscr{F}$ imply $\operatorname{Prob}\left(\|P x\|_{1}>\varepsilon / 2 M\right) \leqq \delta$. Let $Q \geqq P_{0}$. If $B$ is an operator such that $0<\|B\| \leqq M$ then

$$
\begin{aligned}
\operatorname{Prob}\left(\left|\|Q B x\|_{1}-\left\|P_{0} B x\right\|_{1}\right|>\varepsilon / 2\right) & \leqq \operatorname{Prob}\left(\left\|\left(Q-P_{0}\right) B x\right\|_{1}>\varepsilon / 2\right) \\
& \leqq \operatorname{Prob}\left(\left\|\left(Q-P_{0}\right) x\right\|_{1}>\varepsilon / 2\|B\|\right) \\
& \leqq \operatorname{Prob}\left(\left\|\left(Q-P_{0}\right) x\right\|_{1}>\varepsilon / 2 M\right) \\
& \leqq \delta .
\end{aligned}
$$

Letting $Q \rightarrow I$ we get $\operatorname{Pr}\left(\left|\|B x\|_{1}^{\tilde{1}}-\left\|P_{0} B x\right\|_{1}\right|>\varepsilon\right) \leqq \delta$. Hence

$$
\begin{aligned}
& \operatorname{Prob}\left(\left|\|A x\| \tilde{1}-\left\|A_{\alpha} x\right\| \tilde{1}\right|>3 \varepsilon\right) \\
& \quad \leqq \operatorname{Prob}\left(\left|\|A x\| \tilde{1}-\left\|P_{0} A x\right\|_{1}\right|>\varepsilon\right)+\operatorname{Prob}\left(\left\|P_{0}\left(A-A_{\alpha}\right) x\right\|_{1}>\varepsilon\right) \\
& \quad+\operatorname{Prob}\left(\left\|P_{0} A_{\alpha} x\right\|_{1}-\left\|A_{\alpha} x\right\|_{1}>\varepsilon\right) \\
& \leqq 2 \delta+\operatorname{Prob}\left(\|x\| \tilde{1}>\varepsilon /\left\|P_{0}\left(A-A_{\alpha}\right)\right\|\right) .
\end{aligned}
$$

Since $P_{0}$ is finite dimensional and $\left\|P_{0}\left(A-A_{\alpha}\right)\right\|=\left\|\left(A^{*}-A_{\alpha}^{*}\right) P_{0}\right\|$ it follows easily that $\left\|P_{0}\left(A-A_{\alpha}\right)\right\| \rightarrow 0$ as $\alpha \rightarrow \infty$ and therefore the last term of the last inequality goes to zero as $\alpha$ increases. Thus

$$
\operatorname{Prob}\left(\left|\|A x\|_{1}-\left\|A_{\alpha} x\right\|_{1}\right|>3 \varepsilon\right) \leqq 3 \delta \text { for sufficiently large } \alpha .
$$

REMARK. The appearance of adjoints in the statement of Corollary 5.1 is necessary. Thus if $x_{1}, x_{2}, \cdots$ is an O.N. basis of $H$, if $A_{n} x_{j}=x_{1}$ for $j=n$ and equals 0 for $j \neq n$ and if $\|x\|_{n}=\left|\left(x, x_{n}\right)\right|$ then $A_{n} \rightarrow 0$ strongly and $\left\|A_{n}\right\| \leqq 1$ but $\left\|A_{n} x\right\|_{1}=\|x\|_{n}$ does not converge to zero in probability since all the $\|x\|_{n}$ have the same distribution function.

Corollary 5.2. If $P_{\alpha}$ and $P_{\beta}$ are two nets of finite dimensional projections 
each converging to the identity and $\|x\|_{1}$ is a measurable pseudo-norm then $\|x\| \tilde{1}=\lim$ in prob $_{\alpha \rightarrow \infty}\left\|P_{\alpha} x\right\|_{1}$. Furthermore $\left\|\left(P_{\alpha}-P_{\beta}\right) x\right\| \tilde{i}$ converges to zero in probability as $\alpha, \beta \rightarrow \infty$.

Proof. The corollary follows from Corollary 5.1.

COROLlaRY 5.3. If $\left\{P_{\alpha}\right\}_{\alpha \in D}$ is a net of finite dimensional projections converging strongly to the identity and if $f$ is u.c.n. 0 in $H_{m}$ then $\lim$ in prob ${ }_{\alpha \rightarrow \infty} f \circ P_{\alpha}$ exists and equals $f^{\sim}$.

Proof. It suffices to prove that lim in prob.PEF, PED $_{\text {. }}\left|f \circ P_{\alpha}-f \circ P\right|$ is zero. This may be proven by the same method used in Theorem 2 once we know that

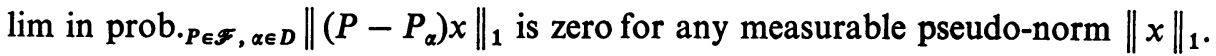
The latter is asserted in Corollary 5.2. q.e.d.

COROLlaRY 5.4. If $\|x\|_{1}$ is a measurable pseudo-norm on $H$ then it is bounded on the unit sphere of $H$. Furthermore if $\|x\|_{n} n=1,2, \cdots$ is a sequence of measurable pseudo-norms such that $\|x\|_{n}^{\tilde{a}}$ approaches zero in probability then $\|x\|_{n}$ converges to zero uniformly on the unit sphere of $H$.

Proof. Let $a_{n}=\operatorname{Prob}\left(\|x\|_{n}>1\right)$. If $\|y\|=1$ then $P x=(x, y) y$ is a projection. Hence keeping $y$ fixed we have

$$
\begin{aligned}
\operatorname{Prob}\left(\left|(x, y)^{\sim}\right|\|y\|_{n}>1\right) & =\operatorname{Prob}\left(\|P x\|_{n}^{\tilde{}}>1\right) \\
& \leqq \operatorname{Prob}\left(\|x\|_{n}^{\sim}>1\right) \\
& \leqq a_{n} .
\end{aligned}
$$

Now the function $x \rightarrow(x, y)$ has a Gaussian distribution. Let $b_{n}$ be such that $2(2 \pi c)^{-1 / 2} \int_{b_{n}}^{\infty} \exp \left[-t^{2} / 2 c\right] d t=a_{n}$. Then $\|y\|_{n}^{-1} \geqq b_{n}$ i.e. $\|y\|_{n} \leqq b_{n}^{-1}$. Furthermore as $a_{n} \rightarrow 0 b_{n} \rightarrow \infty$ and $b_{n}^{-1} \rightarrow 0$ concluding the proof of the corollary.

COROLlary 5.5. If $f$ is u.c.n. 0 in $H_{m}$ then $f^{\sim}$ is the null function if and only if $f$ is identically zero on $H$.

Proof. We note first that if $\|x\|_{1}$ is any measurable pseudo-norm then the function $g(x)=\left\|x-x_{0}\right\|_{1}$ is uniformly continuous in $H_{m}$ and that the associated measurable function $g^{\sim}$ has essential lower bound zero. The latter fact may be proved just as in Theorem 1 by enlarging the projections $P_{j}$ that occur there so as to include $x_{0}$ in their ranges and writing $\left\|\left(P_{k} x\right)-x_{0}\right\|_{1} \leqq\left\|\left(P_{n} x\right)-x_{0}\right\|_{1}$ $+\left\|\left(P_{k}-P_{n}\right) x\right\|_{1}$ and proceeding as therein described.

Now suppose that $f\left(x_{0}\right) \neq 0$. We may assume $f\left(x_{0}\right)=3 a>0$. Since $f$ is u.c.n. 0 in $H_{m}$ there exists a sequence of measurable pseudo-norms converging to zero in probability on the unit ball of each of which $f$ is uniformly continuous in $H_{m}$. By Corollary 5.4 one of these pseudo-norms which we shall denote by $\|x\|_{2}$ has the property that $\left\|x_{0}\right\|_{2} \leqq 1 / 2$. Since $f$ is uniformly continuous on $S=\left\{x:\|x\|_{2}\right.$ 
$\leqq 1\}$ in the topology $H_{m}$ there exists another measurable pseudo-norm $\|x\|_{3}$ such that $|f(x)-f(y)|<a$ whenever $\|x-y\|_{3}<1$ and $x$ and $y$ are in S. Let $\|x\|_{1}=\|x\|_{2}+\|x\|_{3}$. Then $\|x\|_{1}$ is a measurable pseudo-norm. If $\left\|x-x_{0}\right\|_{1}$ $<1 / 2$ then $\|x\|_{3}<1$ and $\left\|x-x_{0}\right\|_{2}<1$ and hence $\left|f(x)-f\left(x_{0}\right)\right|<a$. Put $g(x)=\left\|x-x_{0}\right\|_{1}$. Thus $f(x)>2 a$ whenever $g(x)<1 / 2$. Hence for any $P$ in $\mathscr{F} \operatorname{Prob}(f \circ P>2 a) \geqq \operatorname{Prob}(g \circ P<1 / 2)$. Since $g^{\sim}$ has essential lower bound zero and $g \circ P \rightarrow g^{\sim}$ in probability the right side of the last inequality is bounded away from zero as $P \rightarrow I$ hence so is the left side. It follows that $\operatorname{Prob}\left(f^{\sim}>a\right)>0$.

5. Examples. (1) The function $\|x\|$ is not a measurable pseudo-norm on $H$ for the distribution of the tame function $\|P x\|$ depends only on the rank of the finite dimensional projection $P$.

(2) Mere continuity of a function does not ensure that it will define a measurable function by the prescription given in this paper. In fact the function $f(x)=\exp \left(i\|x\|^{2}\right)$ is uniformly continuous on bounded sets whereas the net $f(P x), P$ in $\mathscr{F}$, does not converge in probability for if $P \geqq Q$ then $|f(P x)-f(Q x)|$ $=\left|\exp \left(i\|(P-Q) x\|^{2}\right)-1\right|$. Thus in order for $f(P x)$ to converge in probabilty it is necessary that for any given $\varepsilon>0$ there exist a projection $P_{0}$ in $\mathscr{F}$ such that $\operatorname{Prob}\left(\left|\exp \left(i\|(P-Q) x\|^{2}\right)-1\right|>\varepsilon\right)<\varepsilon$ whenever $P \geqq Q \geqq P_{0}$. However since the distribution of $\|(P-Q) x\|^{2}$ depends only on the rank of the projection $P-Q$ and not otherwise on $P$ or $Q$ we need only find an $\varepsilon$ for which the last inequality fails when rank $P-Q=1$ in order to conclude that $f(P x)$ does not converge in probability. The existence of such an $\varepsilon$ is clear.

(3) Functions of $\|x\|$ behave badly with respect to the normal distribution since $\|x\|$ itself in no reasonable manner defines a measurable function on $H$ (with respect to the normal distribution). This has been pointed out by Segal [9] and follows from the fact that $\|x\|^{2}=\Sigma_{j}\left(x_{j}, x\right)^{2}$ where $\left\{x_{j}\right\}$ is an O.N. basis. Any measurable function corresponding to $\|x\|^{2}$ would be the sum of an infinite series of independent identically distributed random variables. By Kolmogoroff's three-series theorem such a series diverges with probability one. Now consider the function $f(x)=\exp \left(-\|x\|^{2}\right)$. In this case the net $f(P x)$ converges in probability but the limit is zero even though $f$ is not zero. In fact it is easy to show that it converges to zero in $L_{1}$ for a simple computation yields $E(f(P x))=1 /(1+2 c)^{n / 2}$ where $n=\operatorname{rank} P$. Thus as $P \rightarrow I, E(f(P x)) \rightarrow 0$. By Corollary 5.5 this cannot happen for a nonzero function which is u.c.n. 0 in $H_{m}$.

(4) We shall elaborate here on the relation between certain measurable functions on Wiener space and functions on the space $H=$ real $L^{2}(0,1)$. We denote by $\mathscr{C}$ the Wiener measure space consisting of the continuous functions on $[0,1]$ which vanish at zero. A representative of the normal distribution on $H$ with variance parameter $c=1$ is given by the stochastic integral $n(f)(x)=\int_{0}^{1} f(t) d x(t)$. Here $f$ is in $H, x$ is in $\mathscr{C}$ and $n(f)$ is a random variable on $\mathscr{C}$ defined for almost all $x$ in $\mathscr{C}$. If $G$ is a function on $H$ which is u.c.n. 0 in $H_{m}$ then by Theorem 2 it 
defines a random variable $G^{\sim}=F$ on $\mathscr{C}$. What functions $F$ are obtainable in this way? We shall show that (among others) any function $F$ which is continuous in the sup norm on $\mathscr{C}$ or in the $\operatorname{Lip} \alpha$ norm, $0<\alpha<1 / 2$, is equal to $G^{\sim}$ where $G$ is the function defined by $G(f)=F\left(\int_{0}^{\left({ }^{\circ}\right)} f(s) d s\right)$. $G$ is u.c.n. 0 in $H^{m}$. In proving this we shall also construct some more examples of measurable pseudo-norms.

Let $\mathscr{C}^{\prime}$ be the subset of $\mathscr{C}$ consisting of those absolutely continuous functions $x$ whose derivative $x^{\prime}$ is in $L^{2}(0,1)$. Denote by $t_{j}, j=0,1, \cdots, 2^{m}$ the binary rationals, $t_{j}=j 2^{-m}$. Consider a function $F$ on $\mathscr{C}$ of the form

$$
F(x)=\Phi\left(x\left(t_{1}\right), \cdots, x\left(t_{2^{m}}\right)\right)
$$

where $\Phi$ is a Borel measurable function of $2^{m}$ real variables. Let $G$ be the function on $H$ defined by $G(f)=F\left(\int_{0}^{\left({ }^{\cdot}\right)} f(s) d s\right)$. Thus if $\chi_{t}(s)$ is the characteristic function of $[0, t)$ then $G(f)=\Phi\left(\left(f, \chi_{t}\right), \cdots,\left(f, \chi_{t 2 m}\right)\right)$ and $G$ is a tame funtion on $H$. The corresponding measurable function $G^{\sim}$ on $\mathscr{C}$ is by definition $\tilde{G}(x)$ $=\Phi\left(n\left(\chi_{t_{1}}\right)(x), \cdots, n\left(\chi_{t_{2} m}\right)(x)\right)$. Now $n\left(\chi_{t}\right)(x)=\int_{0}^{1} \chi_{t}(s) d x(s)=x(t)$. Hence $\tilde{G}(x)$ $\Phi\left(x\left(t_{1}\right), \cdots, x\left(t_{2 m}\right)\right)=F(x)$. In other words if we identify $H$ with $\mathscr{C}^{\prime}$ by means of the mapping $f \rightarrow x$ where $x(t)=\int_{0}^{t} f(s) d s$ then the above function $F$ is a tame function on the Hilbert space $\mathscr{C}^{\prime}$ (with inner product $\left.(x, y)=\int_{0}^{1} x^{\prime}(t) y^{\prime}(t) d t\right)$ and the corresponding measurable function is just an extension of $F$ from $\mathscr{C}^{\prime}$ to all of $\mathscr{C}$. In order to extend this relationship between $F$ and $G$ to other functions $F$ we shall first construct some measurable pseudo-norms on $H$.

Consider the function $F_{m}(x)=\sup \left\{\left|x\left(t_{j}\right)\right|: j=1, \cdots, 2^{m}\right\}$. The corresponding function $\|f\|_{m}=F_{m}\left(\int_{0}^{(\cdot)} f(s) d s\right)$ is clearly a tame pseudo-norm on $H$. As $m \rightarrow \infty\|f\|_{m}$ increases to the limit $\|f\|_{0}=\sup \left\{\left|\int_{0}^{t} f(s) d s\right|: 0 \leqq t \leqq 1\right\}$ which is a pseudo-norm on $H$ while the corresponding measurable functions $F_{m}=\|f\|_{m}$ converge everywhere on $\mathscr{C}$ to the function

$$
F(x)=\sup \{|x(t)|: 0 \leqq t \leqq 1\} .
$$

which is well known to have the property that $\operatorname{Prob}(F(x) \leqq \varepsilon)>0$ for all $\varepsilon>0$. Hence $\|f\|_{0}$ is a measurable norm on $H$ by Corollary 4.4 and the corresponding measurable function is $\|f\|_{0}=F(x)$.

Other measurable norms which we shall need are as follows. Let $0<\alpha<1 / 2$. Let $F_{m}^{(\alpha)}(x)=\sup \left\{\left|t_{i}-t_{j}\right|^{-\alpha}\left|x\left(t_{i}\right)-x\left(t_{j}\right)\right|, \quad i \neq j, i, j=0,1, \cdots, 2^{m}\right\}$. The same procedure as in the preceding paragraph may be applied to conclude that the norm $\|f\|^{(\alpha)}=\operatorname{Lip} \alpha$ norm of $x(t)=\int_{0}^{t} f(s) d s$ is a measurable norm on $H$.

Let $F$ be a function on $\mathscr{C}$ which is continuous with respect to the uniform topology on $\mathscr{C}$. Let $G(f)=F\left(\int_{0}^{\left({ }^{\prime}\right)} f(s) d s\right)$. We shall show that $G$ is u.c.n. 0 in $H_{m}$ and that $G^{\sim}=F$. We put $\|x\|_{0}=\sup _{0 \leqq t \leqq 1}|x(t)|$ and $\|x\|_{\alpha}=$ $\sup _{t \neq s}|t-s|^{-\alpha}|x(t)-x(s)|$ for $x$ in $\mathscr{C}$ and $x$ in $\mathscr{C} \cap L i p ~ \alpha$ respectively. We recall that for any fixed $\alpha$ with $0<\alpha<1 / 2$ almost all $x$ in $\mathscr{C}$ are in Lip $\alpha$ and at the same time $S_{k}=\left\{x \in \mathscr{C}:\|x\|_{\alpha} \leqq k\right\}$ is compact with respect to $\|x\|_{0}$. Hence $F$ 
is uniformly continuous on $S_{k}$ with respect to the uniform topology. In particular $F$ is uniformly continuous on $S_{k} \cap \mathscr{C}^{\prime}$ with respect to the uniform topology. In other words we have shown that on the unit ball in $H$ of the measurable norm $k^{-1}\|f\|^{(a)} G$ is uniformly continuous with respect to the norm $\|f\|_{0}$. Since $k^{-1}\|x\|_{\alpha}$ converges to zero a.e. on $\mathscr{C}$ (hence in probability) as $k \rightarrow \infty$ it follows that $G$ is u.c.n. 0 in the topology $H_{m}$. In order to prove that $G^{\sim}=F$ we may apply Corollary 5.3 as follows. Let $P_{m}$ be the projection onto the span of the functions $\chi_{t_{1}}, \cdots, \chi_{t_{2} m}$. Then $\left\{P_{m}\right\}$ is an increasing sequence of projections on $H$ which converges strongly to the identity. Hence $G^{\sim}=\lim$ in prob. $\left(G \circ P_{m}\right)^{\sim}$. For any $x$ in $\mathscr{C}$ let $x^{(m)}$ be the piecewise linear function with vertices at $t_{j}, j=0,1, \cdots, 2^{m}$ which agrees with $x$ at these points. Let $F_{m}(x)=F\left(x^{(m)}\right)$. It is not hard to verify that $F_{m}\left(\int_{0}^{(\cdot)} f(s) d s\right)=G\left(P_{m} f\right)$. Hence $\left(G \circ P_{m}\right)^{\sim}=F_{m}$. As $x^{(m)} \rightarrow x$ in sup norm (and also in Lip $\alpha$ for $x \in \operatorname{Lip} \alpha$ ) it follows that $F_{m} \rightarrow F$ on $\mathscr{C}$ hence in probability. Thus $G^{\sim}=F$.

A similar proof shows that if $F$ is defined on $\mathscr{C} \cap \operatorname{Lip} \beta$ and is continuous in Lip $\beta$ norm where $0<\beta<1 / 2$ then the function $G(f)=F\left(\int_{0}^{(\cdot)} f(s) d s\right)$ is u.c.n. 0 in $H_{m}$ and that $G^{\sim}=F$. One uses the fact that for $\beta<\alpha<1 / 2$ the unit balls of $k^{-1}\|x\|_{\alpha}$ are compact with respect to $\|x\| \beta$.

\section{BIBLIOGRAPHY}

1. T. Bonnesen and W. Fenchel, Theorie der konvexen Körper. Springer, Berlin, 1934.

2. K. O. Friedrichs and H. N. Shapiro, Integration over Hilbert space and outer extensions, Proc. Nat. Acad. Sci. U.S.A. 43 (1957), 336-338.

3. K. O. Friedrichs et al., Integration of functionals, New York University, Mimeographed notes, 1957.

4. R. K. Getoor, On characteristic functions of Banach space valued random variables, Pacific J. Math. 7 (1957), 885-896.

5. L. Gross, Integration and nonlinear transformations in Hilbert space, Trans. Amer. Math. Soc. 94 (1960), 404-440.

6. R. Schatten, $A$ theory of cross spaces, Princeton Univ. Press, Princeton, N.J., 1950.

7. I. E. Segal, Tensor algebras over Hilbert spaces, Trans. Amer. Math. Soc. 81 (1956), 106-134.

8. - Distributions in Hilbert space and canonical systems of operators, Trans. Amer. Math. Soc. 88 (1958), 12-41.

9. - Ergodic subgroups of the orthogonal group on a real Hilbert space, Ann. of Math. 66 (1957), 297-303.

The INSTITUTE for AdVANCEd Study, Princeton, NeW Jersey

YALE UNIVERSITY, New Haven, Connecticut

CORNELl UNIVERSITY, ItHACA, New YoRK 